\title{
Photoablation von biologischem Hartgewebe durch Excimer-Laserstrahlung
}

\author{
R. Jahn, M. Dressel, W. Neu, U. Grzesik, J. Kesper, K. H. Jungbluth
}

Chirurgische Universitätsklinik, Abt. Unfall- und Wiederherstcllungschirurgie (Direktor: Prof. Dr. K. H. Jungbluth) Martinistraße 52, D-2000 Hamburg 20

\section{Zusammenfassung}

Heilungshemmende thermische Gewebsschädigungen nach Lascrschnitten am biologischen Hartgewebe lassen sich nach neueren Untersuchungen mittels eines "athermischen" Abtragprinzips (Photoablation) weitestgehend vermeiden.

In über 400 Einzeltestungen wurden mittels Kombination spezueller Excimerlaser mit Taperfasern die cinzelnen Laserparameter (Pulsbreite, Applikationsenergie, Repetitionsrate, Energıedichte) hunsichtlıch threr Wechselwirkung auf biologisches Hartgewebe untersucht und Bedingungen zur Optimierung der Ablationsraten herausgearbeitet.

\section{Einlcitung}

Schneidversuche am biologıschen Hartgewebe (Knochen) mit den herkömmlichen Lasern aus dem infraroten Spektralbereich (z. B. $\mathrm{CO}_{2}$ - Laser) scheiterten zumeist an der thermischen Schädigung des Gewebes. Es kam zu ciner ausgedehnten Karbonisterung (1,2), die zu Heilungsverzögerungen führte (3, 4).

In den chırurgischen Fachgebieten, die, wie z. B. die Unfallchırurgic vorwiegend Trennverfahren (Sägen, Mcißcln, Bohren) an Knorpel- und Knochengewcbe durchführen, würde cine effiziente Lasertechnologie den Weg zur minimal- invasıven Chırurgie bedeuten. E1n großer Nachteıl der gebräuchlichen mechanischen Instrumente liegt in der zusätzlıchen Traumatısierung eines durch den Unfall bereits geschädigten Gewebes durch z. B. Erschütterungen (Bohren, Meißeln), ausgedehnte Opcrationszugänge (Einbringen des Instrumentariums), Mitverletzung umgebender Strukfuren beim Operieren in Gelenkräumen und beım Abtragen von Verkalkungen in der Nahe von Gefaßen und Nerven.

Zicl der Entwicklung einer geeıgneten Lasertechnologic ist dabeı nicht in crster Linic die absolute Wettbewerbsfähigkcit eınes Lasers in puncto Arbeitsgeschwindıgkeıt ım Vergleich zu Skalpell oder Schere, sondern vielmehr die Verkleınerung der operativen Zugangswege und das atraumatusche Operieren.

Mit der Entdeckung der Photoablation (5) wurde cin Abtragungsvorgang gefunden, der am Beispiel verschiedener industnelier Anwendungen (6,7) höchste Präzisıon aufweıst. Mit cinem kurzgepulsten Hochleistungslaser (Excımerlaser) können härteste Matenaloberflächen (Glas, Keramık, Diamant) crschütterungsfrei und relativ athermisch abgetragen werden. 
Medizinische Versuche, bei denen der Excimerlaser zum Schneiden von Knorpel (8) oder Knochen (3) eingesetzt wurde, waren zunächst wenig erfolgreich. Die Abtragrate war zu gering.

Da die Wechselwirkung zwischen Excimerlaserstrahlung und biologischem Hartgewebe noch nicht eingehend genug untersucht worden ist, zielten die vorliegenden Experimente darauf ab, die einzelnen Lascrparameter zu variieren, um dic Gewebsrcaktionen zu bcobachien.

\section{Matcrial und Methode}

Drci Excimerlaser $(\mathrm{XcCl})$ der Wellenlänge 308nm (Lambda Physik) mit unterschiedlicher Pulsbrette wurden cingesctzt:

EMG I003i (Pulsbrcite 28ns), LPX 605 iCC (Pulsdauer 60ns) und EMG 602 (Pulsdauer 250-300ns).

In Hınblick auf eınen späteren klinischen Einsatz erfolgte die Strahlführung prinzipiell über flexible Taperfasem (Heracus Quarzglas).

Als biologisches Material wurden Rippenknochen und Menısei von frisch geschlachteten Tieren (Schwein, Rind) verwendet.

Die Wechselwirkungen mit dem Gewebe wurden untersucht bei Variation der Applikationsenergie von $20 \mathrm{~mJ}-70 \mathrm{~mJ}$, einer Repetitionsrate von $10-100 \mathrm{~Hz}$ und Energiedichten von $2,13 / \mathrm{cm}^{2}-20 \mathrm{~J} / \mathrm{cm}^{2}$ (Faserkendurchmesser $0,4 \mu \mathrm{m} ; 0,6 \mu \mathrm{m}, 1,0 \mu \mathrm{m}$ ).

Dic Gewebsproben lagen fest auf in einer mit Wasser gefüllten Experimentierschale, die auf eınem optischen Tisch montien war. Darüber war in einer Halterung die Faser arretiert. Mit Hilfe ennes computergesteuerten Schrittmotors wurde während des Schneidens bei konstanter Geschwindigkeit $(2 \mathrm{~mm} / \mathrm{s})$ das Gefäß mit dem Prăparat unter dem austrctenden Laserstrahl hin- und hergeführt. Um enne mechanische Zerstörung der Faser dabei zu vermeiden, wurde ein Anfangsabstand von $0,5 \mathrm{~mm}$ cingestellt. Nach jeweils 8 Querungen konnte das Fascrende um jeweils $0,5 \mathrm{~mm}$ abgesenkt werden. Die Bohrungen erfolgten im Kontaktverfahren.

\section{Ergcbnisse}

\section{Knochenablation}

Die Bedeutung der Pulsbreite erweist sich in den Bereichen niedriger Energiedichten als gerng. Ab ciner Energicdichte von $6 \mathrm{~J} / \mathrm{cm} 2$ steigi die Ablationsrate unter Verwendung des Langpulslasers (300ns) erheblich. Beı ciner Pulsbrcite von 28 ns kommt es frühzeitig zur Zerstönung der Faserendfläche. Der Mittelpulslaser (60ns) werst im Vergleich zum Langpulslaser noch höhere Ablationsraten auf, allerdings werden Repetitionsraten nur bis $40 \mathrm{~Hz}$ toleriert (Langpulslaser über $50 \mathrm{~Hz}$ ). Die erreichten Energiedichten liegen bei $20 \mathrm{~J} / \mathrm{cm} 2$ (Pulsbreite $300 \mathrm{~ns}$ ) und $15 \mathrm{~J} / \mathrm{cm} 2$ (Pulsbrcite $60 \mathrm{~ns}$ ). Dic crreıchte Geschwindigkeit bei Knochenbohrungen beträgt $0,08 \mathrm{~mm} / \mathrm{s}$, entsprechend ciner Ablationsrate von $>2 \mu \mathrm{m} /$ Puls. Karbonisierungen wurden unter dieser Parameterauswahl nucht 
gesehen. Dic Kanten von Bohrungen und Schnitten waren glatt und gratlos.

Ablation von Meniskusgewcbe

Es zeigt sich auch hier cine deutliche Überlegenheit von größeren Pulsbreiten. Die Faserendfäche bleibt länger erhalten. Die Ablationsraten am Menıskusgewebe sind deuthch höher als am Knochen. Es wurde eine Bohrgeschwindigkeit von $2 \mathrm{~mm}$ (maximal $6 \mathrm{~mm}$ ) erreicht. Schnitte über eine Flache von $4 \mathrm{~mm} \times 13 \mathrm{~mm}$ crfolgten in $111 \mathrm{~s}$. Es wurden keine Gewcbeschrumpfung und auch keine Karbonisierung beobachtet. Die Schnittflächen zeigten ein etwas aufgelockertes Gewebe.

\section{Diskussion}

Durch Verlängerung der Haltbarkeitsdauer der Faserendflächen stellt sıch eine deutliche Überlegenheit größerer Pulsbreiten gegenüber dem Standardpuls von 28ns ein.

Die Lochtiefen nehmen mit steıgender Repetitionsrate und Erhöhung der Applikationscnergic (gemessen bis $70 \mathrm{~mJ} 50 \mathrm{~Hz}$ ) zu.

Der Vergleich zwischen Langpulslaser und Mittelpulslaser zeigt, daß beide cinen guten Faserschutz gewähren, die Ablationsrate beim kürzcren Puls (60ns) jedoch höher ist.

Repetitionsraten über $50 \mathrm{~Hz}$ führen bei diesem Laser durch die mechanıschen Rückwirkungen während der Ablation derzeit noch zur Zerstōnung der Faser.

Mit den in dieser Studie angewendeten Laserparametern wurde in keinem der Expenmente der Săttigungsbercich der Ablation erreicht. Die Begrenzung erfolgte stets durch die Zerstörschwelle der Faser.

Daraus ist zu schlußfolgern, daß nach eınigen technischen Verbesserungen mit ciner wetteren Zunahme der Ablatıonsrate zu rechnen ist und sıch auch die Schnittgeschwindigketten für Knochengewebe noch verbessern lassen.

\section{Litcratur}

1. Mockwitz J, Franctzkı M, Prestele K, Schnittversuche mit dem $\mathrm{CO}_{2}$-Laser an Artenen und Knochen

Elcctromedica 4:120-123 (1975)

2. Horch $\mathrm{H} \mathrm{H}$,

Laser- Osteotomie und Anwendungsmöglıchkeiten des Lasers in der oralen Weichteılchırurgie

Habilitationsschnften der Zahn- Mund- und Kıcferheilkunde Quintessenz-Verlags GmbH Berlin 1983

3. Grothucs - Spork M,

Vergleıch der Knochenhellung nach Ságeostcotomie, $\mathrm{CO}_{2}$ - Laserosteotomic und Lxcımer-

Lascrostcotomic am Röhrenknochen des Kanınchens

Inauguraldıssertation Frcıc Universıtät Berlın 1990 


\section{Dinkelaker F,}

Die $\mathrm{CO}_{2}$ - Laser- Osteotomie

Habilitationsschrift aus der Chirurgischen Klinik und Poliklinik der Freien Universität Berlin, Abteilung für Unfall- und Wiederherstellungschirurgie Berlin 1989

\section{Srinivasan $R$,}

Ablation of polymers and biological tissue by ultraviolet lasers

Science 234:559-565 (1986)

6. Excimerlaser für dic Materialbearbeitung-Verfahren und Resultate

Industrial Report Lambda Physik Nr.2 1988

\section{Glass marking}

Highlights Lambda Physik Firmenpublikation Nr. 101986

8. Hohlbach G, Müller K O, Schramm U, Baretton G,

Experimentelle Ergebnisse der Knorpelabrasio mit einem Excimerlaser. Histologische und clektronenmikroskopische Untersuchungen.

Z. Orthop. 127:216-221 (1989) 